Article

\title{
A Click Chemistry Approach towards Flavin-Cyclodextrin Conjugates-Bioinspired Sulfoxidation Catalysts
}

\author{
Petra Tomanová ${ }^{1}$, Jiř́i Šturala ${ }^{1}$, Miloš Buděšínský ${ }^{2}$ and Radek Cibulka ${ }^{1, *}$ \\ Received: 21 September 2015 ; Accepted: 14 October 2015 ; Published: 4 November 2015 \\ Academic Editor: Panayiotis Koutentis \\ 1 Department of Organic Chemistry, University of Chemistry and Technology, Prague, Technická 5, \\ 16628 Prague 6, Czech Republic; peta.tomanova@gmail.com (P.T.); sturalaj@vscht.cz (J.Š.) \\ 2 Institute of Organic Chemistry and Biochemistry AS CR, v.v.i., Flemingovo nám. 2, 16610 Prague 6, \\ Czech Republic; budes@uochb.cas.cz \\ * Correspondence: cibulkar@vscht.cz; Tel.: +420-220-443-688; Fax: +420-220-444-288
}

\begin{abstract}
A click chemistry approach based on the reaction between alkynylflavins and mono(6-azido-6-deoxy)- $\beta$-cyclodextrin has proven to be a useful tool for the synthesis of flavin-cyclodextrin conjugates studied as monooxygenase mimics in enantioselective sulfoxidations.
\end{abstract}

Keywords: click chemistry; cyclodextrin; flavin; monooxygenase; oxidation; sulfoxides; green chemistry

\section{Introduction}

Within the last three decades, flavinium salts have been shown as useful tools for the organocatalytic activation of hydrogen peroxide and oxygen allowing various oxidations to proceed under mild conditions [1-3]. In particular, extensive research on $\mathrm{H}_{2} \mathrm{O}_{2}$-sulfoxidations catalyzed by flavinium salts has resulted in several useful synthetic procedures for mild, chemoselective and stereoselective transformations of sulfides to sulfoxides [4-9]. In these artificial systems, flavinium salts Fl mimic the function of flavin co-factors in monooxygenases [10] via the in situ formation of flavin-4a-hydroperoxide FlOOH, which subsequently oxidizes the substrate (Scheme 1).

Flavoenzymes usually give the corresponding oxygenated products chemoselectively and stereoselectively, which is provided by the stereoselective transfer of activated oxygen from flavin-4a-hydroperoxide to the substrate accommodated in an active site of the enzyme via non-covalent bonding interactions [11-14]. The efficiency of an enzyme is usually sensitive to the modification in its active site as well as to a change in the substrate; both of which can lead to a significant loss of stereoselectivity [12,14-16]. Cyclodextrins have been extensively studied as biomimetic catalysts by utilizing their hydrophobic cavity for non-covalent binding of lipophilic substrates in neat aqueous media [17-19]. Moreover, analogously to an enzyme active site, the cyclodextrin cavity offers a chiral environment for asymmetric transformations [20-25].

Souza and coworkers investigated artificial enzymes based on flavin and cyclodextrin moieties linked together with ether or ester functionality. The conjugates were tested in oxidation of mercaptans, $\mathrm{NADH}^{+}$models and in photooxidation of benzyl alcohols [26-28]. Recently, we have designed sulfoxidation catalysts 1 and 2 (Figure 1) containing a redox-active flavinium "co-factor" and chiral substrate-binding site made using $\alpha$ - or $\beta$-cyclodextrin; both parts were attached by amide bonds [29-31]. The efficiency of these biomimetic catalysts was shown to depend strongly on the structure of the "co-factor", the relative position of both parts of the catalyst as well as the size of the cyclodextrin cavity. The best sulfoxidation catalysts were found to be alloxazinium- $\beta$-cyclodextrin 
conjugates $1 \mathrm{a}$ and $\mathbf{1 b}$, which achieved an ee of up to $91 \%$ for tert-butyl methyl sulfide, $80 \%$ ee for aromatic sulfides and up to $64 \%$ ee for aliphatic sulfides with only $1 \mathrm{~mol} \%$ catalyst in neat aqueous media. It was noteworthy that a small change in the catalyst structure such as turning the alloxazine relative to the cyclodextrin cavity (see catalyst 2) caused the stereoselectivity of the reaction to be completely lost [31].<smiles></smiles>

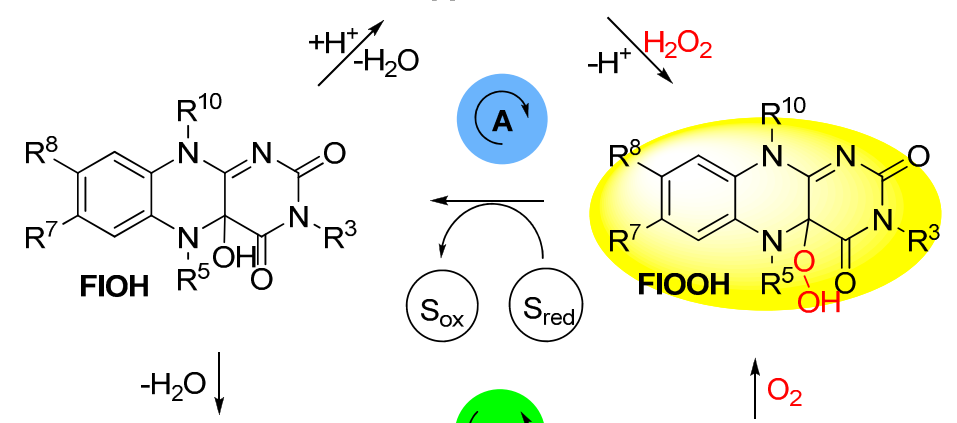
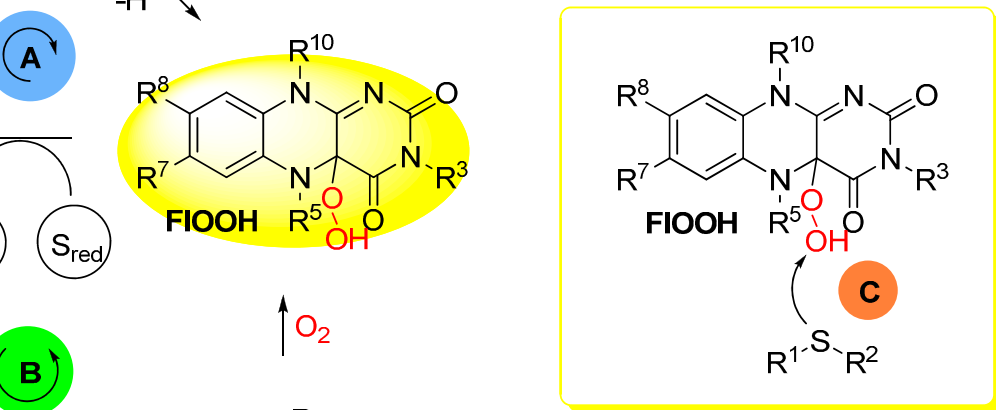<smiles>[R]n1c2nc(=O)[nH]c(=O)c-2nc2cc(C)c(C)cc21</smiles><smiles>[R]N1c2cc(C)c(C)cc2Nc2c1[nH]c(=O)[nH]c2=O</smiles>

Scheme 1. Formation of flavin-4a-hydroperoxide FlOOH in $\mathrm{H}_{2} \mathrm{O}_{2}$-sulfoxidations catalyzed by flavinium salts $\mathbf{F l}\left(\mathrm{R}^{5}=\mathrm{Et}\right)(\mathrm{A})$ and in sulfoxidations taking place in class $\mathrm{B}$ monooxygenases $\left(\mathrm{R}^{5}=\mathrm{H}\right)(\mathbf{B})$. Oxidation of sulfides by FlOOH takes place by electrophilic mechanism (C), i.e., by an attack of sulfur to electrophilic hydroperoxy group oxygen.
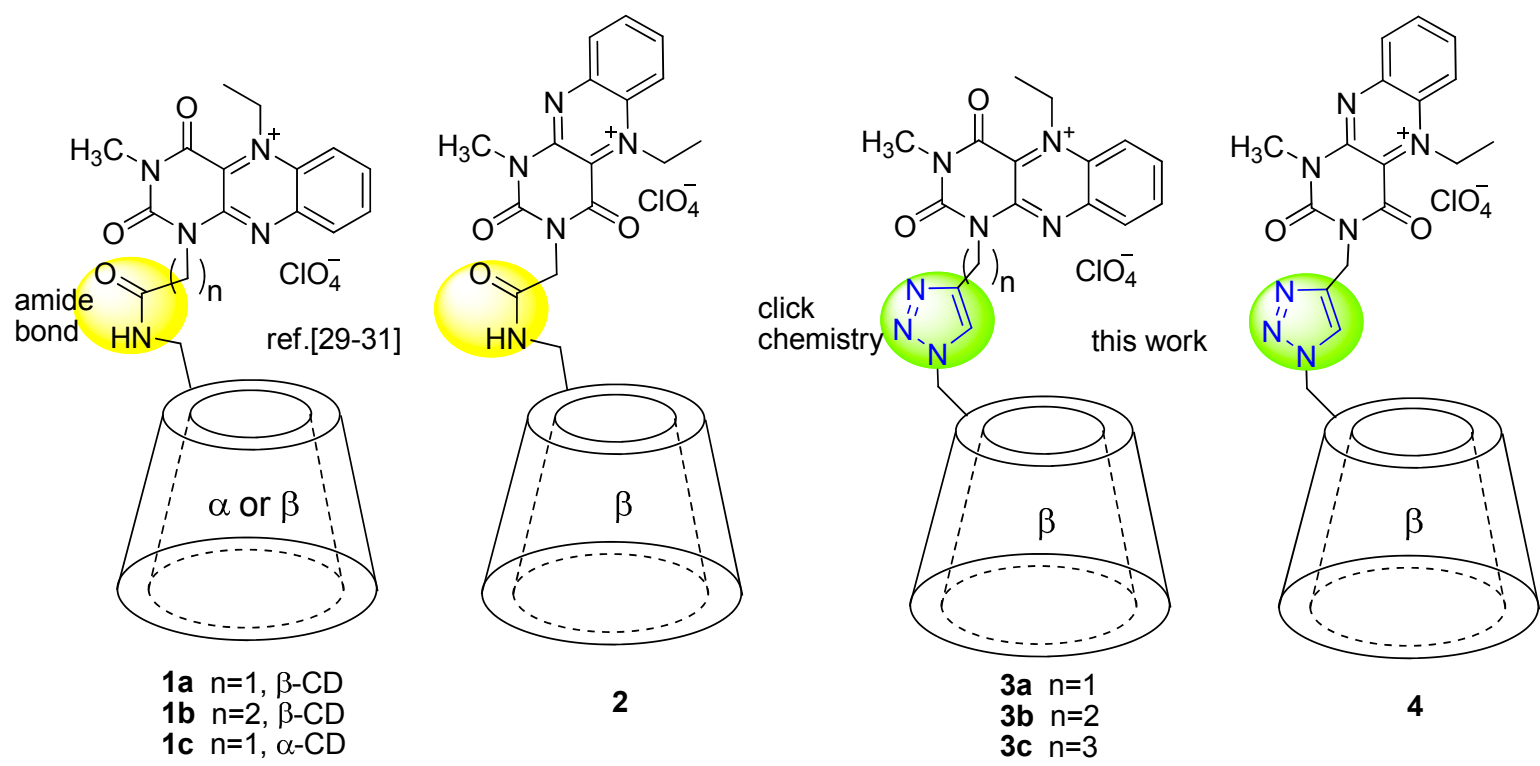

Figure 1. Design of flavin-cyclodextrin conjugates. 
Herein, we report a new synthetic approach towards flavin-cyclodextrin conjugates based on click chemistry, specifically the copper-catalyzed azide-alkyne [3+2] cycloaddition (CuAAC) [32-35]. We used the same co-factor (alloxazine) and cavity ( $\beta$-cyclodextrin) as in $\mathbf{1}$, to prove if the CuAAC methodology is compatible with the alloxazine unit since although click chemistry is well established among cyclodextrins $[34,36]$, its use among flavins is still limited to only one isoalloxazine example $[37,38]$.

\section{Results and Discussion}

\subsection{Synthesis of Conjugates 3 and 4}

The strategy used towards the synthesis of new catalysts 3 and 4 corresponded to the synthetic pathway used for derivatives $\mathbf{1}$ and $\mathbf{2}$ (see Scheme 2). The synthetic route started with the preparation of flavin and cyclodextrin subunits functionalized with suitable groups for interconnecting both parts followed by the transformation of the neutral alloxazine into the corresponding alloxazinium moiety. Alkylation/quaternization of the N5 nitrogen is essential for the flavins to be active in the artificial oxygenations. Amide coupling of mono(6-amino-6-deoxy)- $\beta$-cyclodextrin with the corresponding flavin carboxylic acid used in the synthesis of $\mathbf{1}$ and $\mathbf{2}$ was replaced by a CuAAC between mono(6-azido-6-deoxy)- $\beta$-cyclodextrin 7 and an alloxazine possessing a terminal triple bond in its side chain. 1-(Alkynyl)-3-methylalloxazine 5 and 1-methyl-3-propargylalloxazine 6 were prepared by alkylation of 3-methylalloxazine or 1-methylalloxazine, respectively, with a triple-bond containing alkylating agent. Mono(6-azido-6-deoxy)- $\beta$-cyclodextrin 7 was readily available using standard procedures starting from commercial $\beta$-cyclodextrin [39].<smiles>C#CCn1c(=O)n(C)c(=O)c2nc3ccccc3nc21</smiles><smiles>C#CCn1c(=O)c2nc3ccccc3nc2n(C)c1=O</smiles>

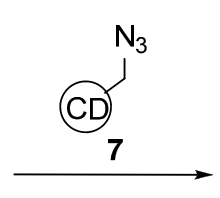

Method A or B
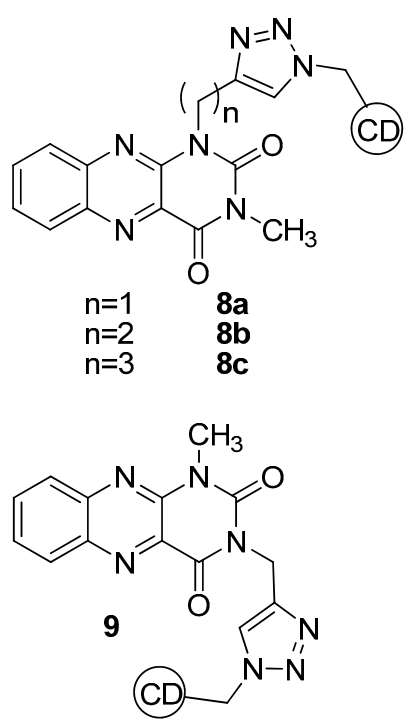

1. $\mathrm{H}_{2}, \mathrm{CH}_{3} \mathrm{CHO}, \mathrm{HClO}_{4}$, ethanol, $\mathrm{H}_{2} \mathrm{O}$

2. $\mathrm{O}_{2}, \mathrm{HClO}_{4}$ 3, 4

Scheme 2. Synthesis of catalysts 3 and 4 using click chemistry approach. Method A: CuI, $\mathrm{N}, \mathrm{N}$-diisopropylethylamine, DMF; method B: TBTA, sodium ascorbate, $\mathrm{CuSO}_{4}, \mathrm{DMF}$.

The preliminary tests of the CuAAC reaction between 3-methyl-1-propargylalloxazine 5a and the model azides (benzylazide or $p$-tolylazide) gave the corresponding triazoles in excellent yield without decomposition of the alloxazine skeleton using conventional reaction conditions [32-35], i.e., copper iodide and Hünig's base in $N, N$-dimethylformamide (DMF) (see Supplementary Materials). Therefore, this method (A) was applied for the cycloaddition of alkynylalloxazines $\mathbf{5}$ and $\mathbf{6}$ with azide 7. In all cases, the corresponding conjugates were formed in moderate yield with the exception of derivative $\mathbf{8 b}$, which was not formed using method A despite great effort (Table 1). Lower yields of conjugates could be a result of redox activity of flavin moiety supporting undesired 
oxidation of $\mathrm{Cu}(\mathrm{I})$ during cycloaddition with less reactive azidocyclodextrine 7 . Therefore we modified reaction conditions using copper sulfate, sodium ascorbate (to produce $\mathrm{Cu}(\mathrm{I})$ in-situ) and the tris[(1-benzyl-1H-1,2,3-triazol-4-yl)methyl]amine (TBTA) ligand which is known to stabilize $\mathrm{Cu}(\mathrm{I})$ towards disproportionation and oxidation (method B) [40]. Under these conditions, $\mathbf{8 b}$ was formed in good yield. Method B even gave the conjugates $\mathbf{8}$ and $\mathbf{9}$ in substantially higher yields, regardless of the position of the alkynyl group on the alloxazine subunit and the length of the spacer (Table 1). Importantly, the triazole spacer tolerated the subsequent reaction steps, which led to the transformation of the neutral alloxazine to alloxazinium moiety, i.e., the introduction of an ethyl group via reductive amination and oxidation, which proceeded in a quantitative yield in all cases.

Table 1. Click reaction between flavins 5 or 6 and azidocyclodextrin $7^{\text {a }}$.

\begin{tabular}{ccccc}
\hline Entry & Alloxazine & Conjugate & Method & Yield \\
\hline 1 & $\mathbf{5 a}$ & $\mathbf{8 a}$ & $\mathrm{A}$ & 68 \\
2 & $\mathbf{5 a}$ & $\mathbf{8 a}$ & $\mathrm{B}$ & 82 \\
3 & $\mathbf{5 b}$ & $\mathbf{8 b}$ & $\mathrm{A}$ & $-\mathrm{b}$ \\
4 & $\mathbf{5 b}$ & $\mathbf{8 b}$ & $\mathrm{B}$ & 58 \\
5 & $\mathbf{5 c}$ & $\mathbf{8 c}$ & $\mathrm{A}$ & 23 \\
6 & $\mathbf{5 c}$ & $\mathbf{8 c}$ & $\mathrm{B}$ & 91 \\
7 & $\mathbf{6}$ & $\mathbf{9}$ & $\mathrm{A}$ & 34 \\
8 & $\mathbf{6}$ & $\mathbf{9}$ & $\mathrm{B}$ & 64 \\
\hline
\end{tabular}

${ }^{a}$ For procedures, see Experimental Section; ${ }^{b}$ Product formation was not observed.

\subsection{Catalytic Activity}

To demonstrate the activity of the modified biomimetic systems in a catalytic oxidation, the prepared conjugates were subjected to preliminary screening in the enantioselective sulfoxidation of alkyl methyl sulfides and aryl methyl sulfides using hydrogen peroxide as a model reaction. The oxidation was performed in a buffered aqueous medium under the reaction conditions recently used in reactions catalyzed by analogous conjugates bearing an amide linker. Among the conjugates bearing a triazole spacer, the efficiency in the enantioselective sulfoxidations decreased with an increase in the spacer length and by "turning" the alloxazine moiety going from 3 to 4 (see Supplementary Materials). In Table 2, the reaction conversions and enantioselectivities of the sulfoxidations catalyzed by $\mathbf{3 a}$, the most efficient conjugate with triazole linker, are compared with the catalytic activity of $\mathbf{1} \mathbf{b}$, one of the most efficient conjugate from "amide" series with the same length of spacer. The reaction conversions of the non-catalyzed oxidation reactions (blanks) are given for information. The observed enantioselectivities obtained using 3a were lower than those found when using $\mathbf{1 b}$ but still comparable or even better than those obtained using other flavin-cyclodextrin conjugates or other chiral flavinium catalysts [7,9,29-31]. Interestingly, the enantioselectivities obtained using 3a were relatively high with difficult substrates such as benzyl, hexyl and butyl methyl sulfides, which are usually oxidized with low enantioselectivities. The lower enantioselectivities compared to $\mathbf{1 b}$ are probably a result of the lower rate of reaction found when using $\mathbf{3 a}$, which is demonstrated by the lower reaction conversions obtained after $1 \mathrm{~h}$; while complete conversion was observed after $60 \mathrm{~min}$ when using catalyst 1 [29-31], the reaction was not finished when using catalyst 3a. This fact causes that the non-stereoselective oxidation of the substrate with hydrogen peroxide (blank) competes significantly with the reaction catalyzed by 3a. This was in accordance with the observation that a higher catalyst loading increased the enantioselectivity of the reactions up to $50 \%$ ee in the case of substrates, which are susceptible to non-catalyzed oxidation (e.g., $t$ - $\mathrm{BuSCH}_{3}$ or $\mathrm{BuSCH}_{3}$ ). 
Table 2. $\mathrm{H}_{2} \mathrm{O}_{2}$-sulfoxidations catalyzed by conjugate $\mathbf{3 a}$ and comparison with data for $\mathbf{1} \mathbf{b}$ and non-catalyzed reaction ${ }^{\text {a }}$.

\begin{tabular}{|c|c|c|c|}
\hline \multirow{2}{*}{ Sulfide } & \multicolumn{2}{|c|}{ Conversion $^{b}(\%) / \mathrm{ee}^{\mathrm{c}}(\%)$} & \multirow[t]{2}{*}{ Conversion of Blank ${ }^{b, d}(\%)$} \\
\hline & $3 a$ & $1 b^{e}$ & \\
\hline$n-\mathrm{C}_{4} \mathrm{H}_{9} \mathrm{SCH}_{3}$ & $\begin{array}{c}\text { quant. } \mathrm{f} / 33 \\
\text { quant. } \mathrm{f} / 50 \mathrm{~g}\end{array}$ & - & 97 \\
\hline$n-\mathrm{C}_{6} \mathrm{H}_{13} \mathrm{SCH}_{3}$ & $42 / 39$ & $92 / 64$ & 10 \\
\hline$n-\mathrm{C}_{8} \mathrm{H}_{17} \mathrm{SCH}_{3}$ & $9 / 11$ & $44 / 29$ & 2 \\
\hline$n-\mathrm{C}_{10} \mathrm{H}_{21} \mathrm{SCH}_{3}$ & $34 / 10$ & $99 / 0$ & 0 \\
\hline$t-\mathrm{C}_{4} \mathrm{H}_{9} \mathrm{SCH}_{3}$ & $\begin{array}{l}\text { quant. }{ }^{f} / 13 \\
\text { quant. }{ }^{f} / 30 \mathrm{~g}\end{array}$ & $98 / 86$ & 98 \\
\hline$c-\mathrm{C}_{6} \mathrm{H}_{11} \mathrm{SCH}_{3}$ & $77 / 26$ & $92 / 80$ & 39 \\
\hline $\mathrm{BnSCH}_{3}$ & $59 / 35$ & $92 / 58$ & 20 \\
\hline$p$-TolylSCH${ }_{3}$ & $36 / 26$ & $96 / 69$ & 4 \\
\hline $\mathrm{PhSCH}_{3}$ & $38 / 20$ & $70 / 36$ & 2 \\
\hline
\end{tabular}

a Conditions: substrate (0.1 mmol), $\mathrm{H}_{2} \mathrm{O}_{2}$ (2.3 equiv.), phosphate buffer $\mathrm{pH} 7.5,25^{\circ} \mathrm{C}$, catalyst loading $1 \mathrm{~mol}$ $\%$ (related to the substrate) if not stated otherwise; vigorous shaking for $1 \mathrm{~h} ;{ }^{\mathrm{b}}$ conversion determined by ${ }^{1} \mathrm{H}-\mathrm{NMR} ;{ }^{\mathrm{c}}$ ee determined by HPLC on a chiral stationary phase (see Supplementary Material for details);

${ }^{\mathrm{d}}$ oxidations without catalyst; ${ }^{\mathrm{e}}$ data from ref. [30]; ${ }^{\mathrm{f}}$ quantitative conversion; ${ }^{\mathrm{g}} 5 \mathrm{~mol} \%$ of the catalyst.

The results show the replacement of amide by triazole spacer influences the catalytic activity of flavin-cyclodextrin conjugates. Most probably higher hydrogen bonding ability of amide function compared to triazole could positively influence the efficiency of amide type catalysts $\mathbf{1}$. Hydrogen bonds could (i) increase the intrinsic reactivity of hydroperoxide function in flavin-4a-hydroperoxide during oxygen transfer to a substrate (see Figure S6 in Supporting Materials for illustration) [41]; and (ii) affect relative arrangement of flavin and cyclodextrin subunits. Nevertheless, as shown recently [31], the catalytic activity of flavin-cyclodextrin conjugates is very sensitive on only minor changes in their structure and thus, further studies are necessary to fully understand the structure activity relationship.

Although flavin-cyclodextrin conjugates do not achieve enantioselectivities exhibited by enzymes (see Table 3), they seem to be promising biomimetic catalysts of sulfoxidations. They provide the oxidations within a relatively short time and in contrast to enzymes, they usually work at higher substrate concentrations. Biocatalytic systems with monooxygenases are more complicated as they require regeneration of NADPH. Cofactor regeneration is usually provided by glucose-6-phosphate dehydrogenase using glucose-6-phosphate as sacrificial reductant. On the other hand biocatalytic systems use oxygen as stoichiometric oxidant, which, advantageously, avoids any background (non-stereoselective) oxidation without participation of the catalyst since oxygen itself is not reactive enough to oxidize most sulfides under mild conditions. Undesired non-catalyzed oxidation unfortunately occurs in oxidations with hydrogen peroxide (see blank experiments in Table 2) thus decreasing overall stereoselectivity. Probably, use of oxygen in place of hydrogen peroxide in oxidations with flavin-cyclodextrin conjugates, analogously to enzymes (combined with a sacrificial reducing agent [2]), would be a way to eliminate some background non-selective oxidations. The other way to improve our catalytic systems could be to optimize the solvent conditions or to increase the reactivity of flavin subunit by introduction of a strong electron-withdrawing group to flavin benzene ring [6]. 
Table 3. Comparison of the catalytic activity of flavin-cyclodextrin conjugates 1a and 3a with enzymes in sulfoxidations of selected sulfides.

\begin{tabular}{|c|c|c|c|c|c|c|}
\hline \multirow{2}{*}{ Catalyst } & \multirow{2}{*}{$\begin{array}{l}\text { Concentration of } \\
\text { Substrate (mM) }\end{array}$} & \multirow{2}{*}{$\begin{array}{l}\text { Reaction } \\
\text { Time (h) }\end{array}$} & \multicolumn{4}{|c|}{ Conversion $(\%) / e e(\%)$} \\
\hline & & & $n-\mathrm{C}_{4} \mathrm{H}_{9} \mathrm{SCH}_{3}$ & $\mathrm{PhSCH}_{3}$ & $p$-TolylSCH 3 & $t-\mathrm{C}_{4} \mathrm{H}_{9} \mathrm{SCH}_{3}$ \\
\hline $1 a^{a}$ & 120 & 1 & - & $93 / 64$ & $99 / 80$ & $99 / 88$ \\
\hline $3 a^{b}$ & 120 & 1 & quant. ${ }^{\mathrm{e}} / 50$ & - & - & quant. $^{\mathrm{e}} / 30$ \\
\hline HAPMO $^{c}$ & $10-20$ & 24 & $99 / 99^{\mathrm{f}}$ & $96 / 99$ & $77 / 99$ & - \\
\hline CHMO $^{d}$ & 35 & overnight & - & $88 / 99$ & $94 / 37$ & $98 / 99$ \\
\hline
\end{tabular}

${ }^{a} 1 \mathrm{~mol} \mathrm{\%}$, data from ref. [30]; ${ }^{\mathrm{b}} 5 \mathrm{~mol} \%$; $^{\mathrm{c}}$ Recombinant 4-hydroxyacetophenon monooxygenase from Pseudomonas fluorescens ACB, $1 \mathrm{U} / \mathrm{mL}$, data from ref. [42]; ${ }^{\mathrm{d}}$ Cyclohexanone monooxygenase from Acinetobacter calcoaceticus, $0.5 \mathrm{U} / \mathrm{mL}$, data from ref. [15]; ${ }^{\mathrm{e}}$ quantitative conversion; ${ }^{\mathrm{f}}$ data from ref. [43].

\section{Experimental Section}

\subsection{General Information}

NMR spectra were obtained on Varian Mercury Plus $300\left({ }^{1} \mathrm{H}\right.$ at $299.9 \mathrm{MHz},{ }^{13} \mathrm{C}$ at $\left.75.5 \mathrm{MHz}\right)$ (Varian, Palo Alto, CA, USA), Agilent 400-MR DDR2 $\left({ }^{1} \mathrm{H}\right.$ at $399.9 \mathrm{MHz},{ }^{13} \mathrm{C}$ at $100.6 \mathrm{MHz}$ ) (Agilent Technologies, Santa Clara, CA, USA), Bruker AVANCE $500\left({ }^{1} \mathrm{H}\right.$ at $500.1 \mathrm{MHz},{ }^{13} \mathrm{C}$ at $\left.125.8 \mathrm{MHz}\right)$ (Bruker Bioscience, Billerica, MA, USA) and Bruker AVANCE $600\left({ }^{1} \mathrm{H}\right.$ at $600.1 \mathrm{MHz},{ }^{13} \mathrm{C}$ at $\left.150.9 \mathrm{MHz}\right)$ spectrometers (Bruker Bioscience). Mass spectra were acquired using ES ionization on LTQ Orbitrap XL spectrometer (ThermoFisher Scientific Inc., Waltham, MA, USA). FT-IR spectra were measured on Nicolet 6700 equipped with ATR accessory (ThermoFisher Scientific Inc.), UV-VIS spectra on Varian Cary 50 spectrometer (Varian). Enantiomeric excess was determined by HPLC on columns with chiral stationary phase equipped with UV detector (wavelength $254 \mathrm{~nm}$ and $215 \mathrm{~nm}$; Ingos, Prague, Czech Republic). TLC and RP-TLC chromatographies were performed with percoated Silica Gel DC Alufolien Kieselgel $60 \mathrm{~F}_{254}$ and 60 RP-18 $\mathrm{F}_{254}$ plates from Merc (Merck-Schuchardt, Hohenbrunn, Germany). Melting points were determined on Boetius microscopic apparatus (Hebenstreit-Rapido $\mathrm{GmbH}$, Radebeul, Germany). Optical rotation was measured on Perkin-Elmer polarimeter 241 (PerkinElmer, Akron, OH, USA).

Alkylating agents 4-bromobut-1-yne [44] and 5-bromopent-1-yne [45-47], and alloxazines 3-methylalloxazine [48] and 1-methylalloxazine [31] were prepared according to previously published procedures.

\subsection{Synthesis of Conjugates}

\subsubsection{Alkylation of 3-Methylalloxazine}

General Procedure

Substituted alloxazine was dissolved in dry $N, N$-dimethylformamide (DMF) and 2 equivalents of alkynylbromide and 5 equivalents of potassium carbonate were added. Reaction mixture was stirred under nitrogen atmosphere for $24 \mathrm{~h}$. The reaction was followed by TLC (chloroform/methanol 100:3). Solid salts were filtered off and solvent was evaporated in vacuo. Residue was dissolved in dichloromethane and washed with water. Water layer was separated and extracted with dichloromethane. Collected organic layers were washed with water and dried with sodium sulfate. After evaporation of solvents, solid product was dried in vacuo.

3-Methyl-1-propargylalloxazine (5a): Synthesis according to general procedure with 3-methylalloxazine $(0.5 \mathrm{~g}, 2.2 \mathrm{mmol})$, propargylbromide $(0.7 \mathrm{~g}, 4.4 \mathrm{mmol})$ and potassium carbonate $(1.5 \mathrm{~g}, 11.0 \mathrm{mmol}) .5 \mathrm{a}$ was obtained as yellow solid (0.5 g, 79\%). M.p. $241-244{ }^{\circ} \mathrm{C} .{ }^{1} \mathrm{H}-\mathrm{NMR}\left(300 \mathrm{MHz}, \mathrm{CDCl}_{3}\right) \delta 8.37$ (dd, $J=8.4,1.0 \mathrm{~Hz}, 1 \mathrm{H}), 8.10(\mathrm{dd}, J=8.4 \mathrm{~Hz}, 1 \mathrm{H}), 7.93(\mathrm{ddd}, J=8.5,6.8,1.5 \mathrm{~Hz}, 1 \mathrm{H}), 7.80(\mathrm{ddd}, J=8.4,6.9$, $1.4 \mathrm{~Hz}, 1 \mathrm{H}), 5.26(\mathrm{~d}, J=2.4 \mathrm{~Hz}, 2 \mathrm{H}), 3.63(\mathrm{~s}, 3 \mathrm{H}), 2.23(\mathrm{t}, J=2.5 \mathrm{~Hz}, 1 \mathrm{H}),{ }^{13} \mathrm{C}-\mathrm{NMR}\left(75 \mathrm{MHz}, \mathrm{CDCl}_{3}\right)$ 
$\delta 159.28,149.60,143.62,142.87,140.04,133.77,130.52,129.23,127.72,71.18,31.68,28.98$. HRMS-ESI ${ }^{+}$ $m / z:[\mathrm{M}+\mathrm{Na}]^{+}$calcd for $\mathrm{C}_{14} \mathrm{H}_{10} \mathrm{~N}_{4} \mathrm{NaO}_{2} 289.0696$, found 289.0696 .

1-(But-3-ynyl)-3-methylalloxazine (5b): Synthesis according to general procedure with 3-methylalloxazine $(1.0 \mathrm{~g}, 4.4 \mathrm{mmol})$, 4-brombut-1-yn $(1.1 \mathrm{~g}, 8.8 \mathrm{mmol})$ and potassium carbonate $(3.0 \mathrm{~g}, 22.0 \mathrm{mmol}) . \quad 5 \mathbf{b}$ was obtained $(1.2 \mathrm{~g}, 95 \%)$ as yellow solid. M.p. $260-261{ }^{\circ} \mathrm{C} .{ }^{1} \mathrm{H}-\mathrm{NMR}$ $\left(300 \mathrm{MHz}, \mathrm{CDCl}_{3}\right) \delta 8.39-8.32(\mathrm{~m}, 1 \mathrm{H}), 8.09-8.02(\mathrm{~m}, 1 \mathrm{H}), 7.92(\mathrm{ddd}, J=8.5,6.9,1.5 \mathrm{~Hz}, 1 \mathrm{H}), 7.78$ $(\mathrm{ddd}, J=8.4,6.8,1.4 \mathrm{~Hz}, 1 \mathrm{H}), 4.69(\mathrm{t}, J=7.3 \mathrm{~Hz}, 2 \mathrm{H}), 3.61(\mathrm{~s}, 3 \mathrm{H}), 2.78(\mathrm{td}, J=7.3,2.7 \mathrm{~Hz}, 2 \mathrm{H})$, $1.97(\mathrm{t}, J=2.7 \mathrm{~Hz}, 1 \mathrm{H}),{ }^{13} \mathrm{C}-\mathrm{NMR}\left(75 \mathrm{MHz}, \mathrm{CDCl}_{3}\right) \delta 159.96,150.50,146.94,145.56,143.42,140.41$, $134.19,131.04,129.56,128.14,81.41,70.56,41.09,29.45,17.67$. HRMS-ESI $^{+} m / z:\left[\mathrm{M}+\mathrm{Na}^{+}\right.$calcd for $^{-}$ $\mathrm{C}_{15} \mathrm{H}_{12} \mathrm{~N}_{4} \mathrm{NaO}_{2}$ 303.0858, found 303.0855.

3-Methyl-1-(pent-4-ynyl)alloxazine (5c): Synthesis according to general procedure with 3-methylalloxazine (39 mg, $0.17 \mathrm{mmol})$, 5-brompent-1-yn $(50 \mathrm{mg}, 0.34 \mathrm{mmol}$ ) and potassium carbonate $(118 \mathrm{mg}, 0.85 \mathrm{mmol}) .5 \mathrm{c}$ was obtained $(45 \mathrm{mg}, 90 \%)$ as yellow solid. M.p. $262-264{ }^{\circ} \mathrm{C}$. ${ }^{1} \mathrm{H}-\mathrm{NMR}\left(300 \mathrm{MHz}, \mathrm{CDCl}_{3}\right) \delta 8.35(\mathrm{~d}, J=7.9 \mathrm{~Hz}, 1 \mathrm{H}), 8.04(\mathrm{~d}, J=8.5 \mathrm{~Hz}, 1 \mathrm{H}), 7.95-7.87(\mathrm{~m}, 1 \mathrm{H})$, 7.81-7.72 (m, 1H), $4.60(\mathrm{t}, 2 \mathrm{H}), 3.61(\mathrm{~s}, 3 \mathrm{H}), 2.38(\mathrm{td}, J=6.9,2.5 \mathrm{~Hz}, 2 \mathrm{H}), 2.18-2.02(\mathrm{~m}, 3 \mathrm{H}), 1.95$ $(\mathrm{t}, J=2.7 \mathrm{~Hz}, 1 \mathrm{H}),{ }^{13} \mathrm{C}-\mathrm{NMR}\left(75 \mathrm{MHz}, \mathrm{CDCl}_{3}\right) \delta 150.83,145.28,143.70,140.41,134.06,131.02$, $129.42,128.14,98.52,83.50,68.99,42.26,34.87,29.40,26.46,16.55$. HRMS-ESI $^{+} m / z:[\mathrm{M}]^{+}$calcd $\mathrm{C}_{16} \mathrm{H}_{14} \mathrm{~N}_{4} \mathrm{NaO}_{2}{ }^{+}$317.1014, found 317.1011.

1-Methyl-3-propargylalloxazine (6): Synthesis according to general procedure with 1-methylalloxazine $(0.5 \mathrm{~g}, 2.2 \mathrm{mmol})$, propargylbromide $(0.7 \mathrm{~g}, 4.4 \mathrm{mmol})$ and potassium carbonate $(1.5 \mathrm{~g}, 11.0 \mathrm{mmol}) .6$ was obtained $(0.4 \mathrm{~g}, 69 \%)$ as yellow solid. M.p. $186-187^{\circ} \mathrm{C} .{ }^{1} \mathrm{H}-\mathrm{NMR}\left(300 \mathrm{MHz}, \mathrm{CDCl}_{3}\right) \delta 8.43-8.27$ $(\mathrm{m}, 1 \mathrm{H}), 8.11-7.99(\mathrm{~m}, 1 \mathrm{H}), 7.92(\mathrm{ddd}, J=8.5,6.8,1.5 \mathrm{~Hz}, 1 \mathrm{H}), 7.78(\mathrm{ddd}, J=8.4,6.8,1.5 \mathrm{~Hz}, 1 \mathrm{H})$, $4.97(\mathrm{~d}, J=2.5 \mathrm{~Hz}, 2 \mathrm{H}), 3.85(\mathrm{~s}, J=3.7 \mathrm{~Hz}, 3 \mathrm{H}), 2.26-2.23(\mathrm{~m}, 1 \mathrm{H}) .{ }^{13} \mathrm{C}-\mathrm{NMR}\left(101 \mathrm{MHz}, \mathrm{CDCl}_{3}\right) \delta$ 159.01, 149.96, 145.55, 143.39, 140.07, 134.13, 130.85, 129.69, 129.36, 127.89, 77.58, 71.43, 31.69, 29.70. HRMS-ESI ${ }^{+} m / z:[\mathrm{M}+\mathrm{Na}]^{+}$calcd for $\mathrm{C}_{14} \mathrm{H}_{10} \mathrm{~N}_{4} \mathrm{NaO}_{2} 289.0696$, found 289.0696.

\subsubsection{Click Reactions}

General Procedure A

The mixture of alkynyl alloxazine 5 or 6 and 6-azido-6-deoxy- $\beta$-cyclodextrin 7 (1.2 equiv.) was stirred with copper(I) iodide ( $3 \mathrm{~mol} \%$ ) and $N, N$-diisopropylethylamine ( 2 equiv.) in dry $\mathrm{N}, \mathrm{N}$-dimethylformamide (DMF) at room temperature for $24 \mathrm{~h}$. Conjugate was precipitated after addition of acetone and separated by centrifugation. Crude product was purified by flash chromatography on the column with reversed phase (gradient $\mathrm{MeOH} / \mathrm{H}_{2} \mathrm{O}$ 1:9 $\rightarrow \mathrm{MeOH} / \mathrm{H}_{2} \mathrm{O}$ 2:3). The obtained solid product 8 or 9 was dried in vacuo.

\section{General Procedure B}

The mixture of alkynyl alloxazine 5 or 6 and 6-azido-6-deoxy- $\beta$-cyclodextrin 7 (1.2 equiv.) was stirred with TBTA (tris[(1-benzyl-1H-1,2,3-triazol-4-yl)methyl]amine) (20 mol \%), sodium ascorbate (20 mol \%) and copper(II) sulfate (10 mol \%) in dry $N, N$-dimethylformamide (DMF) at room temperature for $24 \mathrm{~h}$. Conjugate was precipitated after addition of acetone and separated by centrifugation. Crude product was purified by flash chromatography on the column with reversed phase (gradient $\mathrm{MeOH} / \mathrm{H}_{2} \mathrm{O} 1: 9 \rightarrow \mathrm{MeOH} / \mathrm{H}_{2} \mathrm{O}$ 2:3). The obtained solid product 8 or 9 was dried in vacuo.

1-\{[1-(6-Deoxy-ß-cyclodextrin-6-yl)-1H-1,2,3-triazol-4-yl]methyl\}-3-methylalloxazine (8a): $\quad$ Synthesis according to general procedure A for click reactions using 3-methyl-propargylalloxazine $(75 \mathrm{mg}$, $0.28 \mathrm{mmol}$ ), 6-azido-6-deoxy- $\beta$-cyclodextrin ( $390 \mathrm{mg}, 0.34 \mathrm{mmol})$, copper(I) iodide ( $3 \mathrm{mg}, 0.01 \mathrm{mmol}$ ), 
$\mathrm{N}, \mathrm{N}$-diisopropylethylamine $(98 \mu \mathrm{L}, 0.56 \mathrm{mmol})$ and $4 \mathrm{~mL}$ of dry DMF. Product $8 \mathrm{a}(270 \mathrm{mg}, 68 \%)$ was obtained as yellowish powder.

Synthesis according to general procedure $B$ for click reactions using 3-methyl-propargylalloxazine (28 mg, $0.11 \mathrm{mmol})$, 6-azido-6-deoxy- $\beta$-cyclodextrin $(164 \mathrm{mg}$, $0.14 \mathrm{mmol}$ ), TBTA (11 mg, $0.02 \mathrm{mmol})$, sodium ascorbate $(4 \mathrm{mg}, 0.02 \mathrm{mmol})$, copper(II) sulfate (3 mg, $0.01 \mathrm{mmol}), 4 \mathrm{~mL}$ of dry DMF. Product $8 \mathrm{a}(123 \mathrm{mg}, 83 \%)$ was obtained as yellowish powder. M.p. $238-239{ }^{\circ} \mathrm{C}$. ${ }^{1} \mathrm{H}-\mathrm{NMR}$ see Tables S2 and S4, ${ }^{13} \mathrm{C}-\mathrm{NMR}$ see Tables S1 and S3, HRMS-ESI ${ }^{+} m / z:[\mathrm{M}+\mathrm{Na}]^{+}$calcd for $\mathrm{C}_{56} \mathrm{H}_{79} \mathrm{~N}_{7} \mathrm{NaO}_{36}$ 1448.4458, found 1448.4456. $[\alpha]_{D}^{20}=105.2^{\circ}$ $\left(c=0.107 \mathrm{~g} / 100 \mathrm{~mL}, \mathrm{H}_{2} \mathrm{O}\right)$.

1-\{[1-(6-Deoxy- $\beta$-cyclodextrin-6-yl)-1H-1,2,3-triazol-4-yl]ethyl\}-3-methylalloxazine $\quad(\mathbf{8 b}): \quad$ Synthesis according to general procedure B for click reactions using 1-but-3-ynyl-3-methylalloxazine (30 mg, $0.11 \mathrm{mmol}), 6$-azido-6-deoxy- $\beta$-cyclodextrin 7 (164 mg, $0.14 \mathrm{mmol})$, TBTA (11 mg, $0.02 \mathrm{mmol}$ ), sodium ascorbate ( $4 \mathrm{mg}, 0.02 \mathrm{mmol})$, copper(II) sulfate $(3 \mathrm{mg}, 0.01 \mathrm{mmol}), 4 \mathrm{~mL}$ of dry DMF. Product $8 \mathbf{b}(90 \mathrm{mg}, 58 \%)$ was obtained as yellowish powder. M.p. $241-242{ }^{\circ} \mathrm{C} .{ }^{1} \mathrm{H}-\mathrm{NMR}$ see Tables S2 and S4, ${ }^{13} \mathrm{C}$-NMR see Tables S1 and S3, HRMS-ESI ${ }^{+} \mathrm{m} / z$ : [M + Na $]^{+}$calcd for $\mathrm{C}_{57} \mathrm{H}_{81} \mathrm{~N}_{7} \mathrm{NaO}_{36}$ 1462.4615, found 1462.4615. $[\alpha]_{D}^{20}=104.6^{\circ}\left(c=0.108 \mathrm{~g} / 100 \mathrm{~mL}, \mathrm{H}_{2} \mathrm{O}\right)$.

1-\{[1-(6-Deoxy- $\beta$-cyclodextrin-6-yl)-1H-1,2,3-triazol-4-yl]propyl\}-3-methylalloxazine (8c): $\quad$ Synthesis according to general procedure $\mathrm{A}$ for click reactions using 3-methyl-1-pent-4-ynylalloxazine (35 mg, 0.14 mmol), 6-azido-6-deoxy- $\beta$-cyclodextrin 7 (166 mg, $0.14 \mathrm{mmol})$, copper(I) iodide (1 mg, $0.01 \mathrm{mmol}), \mathrm{N}, \mathrm{N}$-diisopropylethylamine $(41 \mu \mathrm{L}, 0.24 \mathrm{mmol})$ and $2 \mathrm{~mL}$ of dry DMF. Product $8 \mathrm{c}$ (40 $\mathrm{mg}, 23 \%$ ) was obtained as yellowish powder.

Synthesis according to general procedure $B$ for click reactions using 3-methyl-1-pent-4-ynylalloxazine ( $35 \mathrm{mg}, 0.12 \mathrm{mmol}$ ), 6-azido-6-deoxy- $\beta$-cyclodextrin 7 (182 mg, $0.15 \mathrm{mmol})$, TBTA $(12 \mathrm{mg}, 0.02 \mathrm{mmol})$, sodium ascorbate $(4 \mathrm{mg}, 0.02 \mathrm{mmol})$, copper(II) sulfate (3 mg, $0.01 \mathrm{mmol}), 4 \mathrm{~mL}$ of dry DMF. Product $8 \mathrm{c}(157 \mathrm{mg}, 91 \%)$ was obtained as yellowish powder. M.p. $272-274{ }^{\circ} \mathrm{C} .{ }^{1} \mathrm{H}-\mathrm{NMR}$ see Tables S2 and S4, ${ }^{13} \mathrm{C}-\mathrm{NMR}$ see Tables S1 and S3, HRMS-ESI ${ }^{+} m / z:[\mathrm{M}+\mathrm{H}]^{+}$calcd for $\mathrm{C}_{58} \mathrm{H}_{84} \mathrm{~N}_{7} \mathrm{O}_{36}$ 1454.4952, found 1454.4958. $[\alpha]_{D}^{20}=112.3^{\circ}$ $\left(c=0.110 \mathrm{~g} / 100 \mathrm{~mL}, \mathrm{H}_{2} \mathrm{O}\right)$.

3-\{[1-(6-Deoxy-ß-cyclodextrin-6-yl)-1H-1,2,3-triazol-4-yl]methyl\}-1-methylalloxazine (9): $\quad$ Synthesis according to general procedure A for click reactions using 1-methyl-3-propargylalloxazine $(25 \mathrm{mg}$, $0.07 \mathrm{mmol})$, 6-azido-6-deoxy- $\beta$-cyclodextrin 7 (130 mg, $0.11 \mathrm{mmol})$, copper(I) iodide (1 mg, $0.01 \mathrm{mmol}), N, N$-diisopropylethylamine $(33 \mu \mathrm{L}, 0.19 \mathrm{mmol})$ and $1 \mathrm{~mL}$ of dry DMF. Yellowish product $9(46 \mathrm{mg}, 34 \%)$ was obtained.

Synthesis according to general procedure $B$ for click reactions using 1-methyl-3-propargylalloxazine ( $56 \mathrm{mg}, 0.21 \mathrm{mmol}$ ), 6-azido-6-deoxy- $\beta$-cyclodextrin 7 (328 mg, $0.29 \mathrm{mmol}$ ), TBTA (22 $\mathrm{mg}, 0.04 \mathrm{mmol})$, sodium ascorbate $(8 \mathrm{mg}, 0.04 \mathrm{mmol})$, copper(II) sulfate (6 mg, $0.02 \mathrm{mmol}), 4 \mathrm{~mL}$ of dry DMF. Product $9(192 \mathrm{mg}, 64 \%)$ was obtained as yellowish powder. M.p. $\quad 288-290{ }^{\circ} \mathrm{C} .{ }^{1} \mathrm{H}-\mathrm{NMR}$ see Tables S2 and S4, ${ }^{13} \mathrm{C}-\mathrm{NMR}$ see Tables S1 and S3, HRMS-ESI ${ }^{+} m / z$ : $[\mathrm{M}+\mathrm{Na}]^{+}$calcd for $\mathrm{C}_{56} \mathrm{H}_{79} \mathrm{~N}_{7} \mathrm{NaO}_{36}$ 1448.4458, found 1448.4434. $[\alpha]_{D}^{20}=90.6^{\circ}$ $\left(c=0.102 \mathrm{~g} / 100 \mathrm{~mL}, \mathrm{H}_{2} \mathrm{O}\right)$.

\subsubsection{Quaternization of Conjugates}

General Procedure

Conjugate 8 or 9 was mixed with acetaldehyde (300 equiv.), palladium on carbon (10\% $w / w)$, ethanol, water and $0.1 \mathrm{M}$ perchloric acid. Reaction mixture was stirred in autoclave for $21 \mathrm{~h}$ under hydrogen atmosphere (0.6 MPa). Palladium was filtered off and organic solvents were evaporated in vacuo. Residue was diluted with water to adjust the volume to exactly $2 \mathrm{~mL}$. 
Despite our great efforts, we were unable to record clear NMR spectra of the quaternized conjugates due to peak broadening as a result of the presence of radical species [29-31]. Nevertheless, in mass spectra only signals of alloxazinium ions were found. UV spectra of all the prepared catalysts showed maxima at approximately 445 and $382 \mathrm{~nm}$, which are characteristic for alloxazinium salts. For details, see Supplementary Materials.

1-\{[1-(6-Deoxy- $\beta$-cyclodextrin-6-yl)-1H-1,2,3-triazol-4-yl]methyl\}-3-methylalloxazinium perchlorate (3a): Synthesis according to general procedure for quarternisation of conjugates using conjugate $8 \mathbf{a}(15 \mathrm{mg}$, $10 \mu \mathrm{mol})$, acetaldehyde $(170 \mu \mathrm{L}, 3 \mathrm{mmol})$, palladium on carbon $(2 \mathrm{mg}, 10 \% \mathrm{w} / w)$, ethanol $(800 \mu \mathrm{L})$, perchloric acid $(800 \mu \mathrm{L}, 0.1 \mathrm{M})$, water $(600 \mu \mathrm{L})$. HRMS-ESI ${ }^{+} m / z:[\mathrm{M}+\mathrm{H}]^{+}$calcd for $\mathrm{C}_{58} \mathrm{H}_{84} \mathrm{~N}_{7} \mathrm{O}_{36}$ 1454.4952, found 1454.4955, UV-Vis $\left(\mathrm{pH}=3.6, c=5.0 \times 10^{-5} \mathrm{~mol} / \mathrm{L}\right): 382 \mathrm{~nm}, 441 \mathrm{~nm}$.

1-\{[1-(6-Deoxy-ß-cyclodextrin-6-yl)-1H-1,2,3-triazol-4-yl]ethyl\}-3-methylalloxazinium perchlorate (3b): Synthesis according to general procedure for quarternisation of conjugates using conjugate $\mathbf{8 b}$ (11 mg, $7 \mu \mathrm{mol})$, acetaldehyde $(120 \mu \mathrm{L}, 2 \mathrm{mmol})$, palladium on carbon $(2 \mathrm{mg}, 10 \% w / w)$, ethanol $(570 \mu \mathrm{L})$, perchloric acid $(570 \mu \mathrm{L}, 0.1 \mathrm{M})$ and water $(430 \mu \mathrm{L})$. HRMS-ESI $^{+} m / z:[\mathrm{M}+\mathrm{H}]^{+}$calcd for $\mathrm{C}_{59} \mathrm{H}_{86} \mathrm{~N}_{7} \mathrm{O}_{36} 1468.5109$, found 1468.5122. UV-Vis $\left(\mathrm{pH}=3.6, c=3.6 \times 10^{-5} \mathrm{~mol} / \mathrm{L}\right): 382 \mathrm{~nm}, 448 \mathrm{~nm}$.

1-\{[1-(6-Deoxy-ß-cyclodextrin-6-yl)-1H-1,2,3-triazol-4-yl)propyl)-3-methylalloxazinium perchlorate (3c): Synthesis according to general procedure for quarternisation of conjugates using conjugate 8c (12 mg, $8 \mu \mathrm{mol})$, acetaldehyde $(130 \mu \mathrm{L}, 2 \mathrm{mmol})$, palladium on carbon $(2 \mathrm{mg}, 10 \% \mathrm{w} / w)$, ethanol $(620 \mu \mathrm{L})$, perchloric acid $(620 \mu \mathrm{L}, 0.1 \mathrm{M})$ and water $(460 \mu \mathrm{L})$. HRMS-ESI ${ }^{+} \mathrm{m} / z:[\mathrm{M}+\mathrm{H}]^{+}$calcd for $\mathrm{C}_{60} \mathrm{H}_{88} \mathrm{~N}_{7} \mathrm{O}_{36} 1482.5265$, found 1482.5272. UV-Vis $\left(\mathrm{pH}=4.1, c=6.2 \times 10^{-5} \mathrm{~mol} / \mathrm{L}\right): 382 \mathrm{~nm}, 448 \mathrm{~nm}$.

3-\{[1-(6-Deoxy-ß-cyclodextrin-6-yl)-1H-1,2,3-triazol-4-yl]methyl\}-1-methylalloxazinium perchlorate (4): Synthesis according to general procedure for quarternisation of conjugates using conjugate 9 (45 mg, $30 \mu \mathrm{mol})$, acetaldehyde $(510 \mu \mathrm{L}, 9 \mathrm{mmol})$, palladium on carbon $(7 \mathrm{mg}, 10 \% \mathrm{w} / w)$, ethanol $(2.4 \mathrm{~mL})$, perchloric acid $(2.4 \mathrm{~mL}, 0.1 \mathrm{M})$ and water $(1.8 \mathrm{~mL})$. HRMS-ESI ${ }^{+} \mathrm{m} / \mathrm{z}:[\mathrm{M}+\mathrm{H}]^{+}$calcd for $\mathrm{C}_{58} \mathrm{H}_{84} \mathrm{~N}_{7} \mathrm{O}_{36}$ 1454.4952, found 1454.4954, UV-Vis $\left(\mathrm{pH}=4.3, c=1.6 \times 10^{-5} \mathrm{~mol} / \mathrm{L}\right): 385 \mathrm{~nm}, 448 \mathrm{~nm}$.

\subsection{Catalytic Oxidations}

Catalytic oxidations of alkyl or aryl methyl sulfides were performed in $1 \mathrm{~mL}$ thick-walled screw-capped vial (Supelco). The reaction mixtures were prepared by addition of sodium-phosphate buffer $(\mathrm{pH}=7.5,0.05 \mathrm{M}, 300 \mu \mathrm{L})$, liquid substrate $\left(3 \times 10^{-5} \mathrm{~mol}\right)$, appropriate volume of catalyst solution ( $1 \mathrm{~mol} \%-5 \mathrm{~mol} \%$ ) and finally aqueous hydrogen peroxide (2.3 mol equiv.). Stirring of the reaction mixture was provided by wrist action shaker $(900 \mathrm{rpm})$ for $1 \mathrm{~h}$ at $25^{\circ} \mathrm{C}$. Then the reaction was quenched by addition of a solution of sodium dithionite in water $(1.4 \mathrm{M}, 170 \mu \mathrm{L})$. The mixture was extracted with $\mathrm{CDCl}_{3}(3 \times 0.5 \mathrm{~mL})$. Collected organic layers were dried with molecular sieves ( $3 \AA$ ) and used for ${ }^{1} \mathrm{H}-\mathrm{NMR}$ determination of the conversion. Enantiomeric excess was determined by HPLC analysis on column with chiral stationary phase of the sample obtained by evaporation of $\mathrm{CDCl}_{3}$ and dissolving a residue in heptane $(0.5 \mathrm{~mL})$.

\section{Conclusions}

We have demonstrated the CuAAC concept is useful for the preparation of flavin-cyclodextrin conjugates. The click chemistry represents alternative approach to the original procedure based on amide coupling and seems to be slightly more advantageous especially by reducing the number of reaction steps and improving the overall yield. Recently, Rotello found the isoalloxazine ring tolerates "click chemistry" when preparing flavin-polymer conjugates [37,38]; we have shown the CuAAC approach was tolerated by alloxazines. Therefore, it seems CuAAC can be used as a general tool for the construction of flavin (isoalloxazin or alloxazine) based supramolecular systems, which can be useful not only in organocatalysis, but also in photocatalysis [3,26-28] and molecular recognition [49]. The new conjugates show lower efficiency in the enantioselective sulfoxidation 
reaction when compared with catalyst $\mathbf{1}$, which possesses an amide linker. However, $\mathbf{3 a}$ is comparable with the other chiral flavin catalysts [1-3] and even overcomes efficiency of flavin conjugates with amide linker in butyl methyl sulfide oxidation achieving ee up to $50 \%$. One should bear in mind that cyclodextrin-based catalysts usually achieve rather moderate enantioselectivities and the results found with conjugate $\mathbf{1}$ are rather exceptional. The extreme sensitivity to small structural changes observed among the flavin-cyclodextrin conjugates is a typical phenomenon that also occurs in the natural enzymes and its elucidation is currently being investigated in our laboratory.

Supplementary Materials: Supplementary materials can be accessed at: http://www.mdpi.com/1420-3049/ 20/11/19667/s1.

Acknowledgments: The authors wish to thank the Czech Science Foundation (Grant No. P207/12/0447) and Ministry of Education, Youth and Sports of the Czech Republic (Specific university research No. 20/2015) for financial support. We thank Jan Zelenka for preparation of samples of conjugates.

Author Contributions: P.T. performed the experiments and analyzed the data, J.Š. contributed to optimization of click chemistry procedure and to results discussion, M.B. measured and analyzed NMR data of conjugates and R.C. contributed to discussion and wrote the paper.

Conflicts of Interest: The authors declare no conflict of interest.

\section{References}

1. Iida, H.; Imada, Y.; Murahashi, S.I. Biomimetic flavin-catalysed reactions for organic synthesis. Org. Biomol. Chem. 2015, 13, 7599-7613. [CrossRef] [PubMed]

2. Cibulka, R. Artificial flavin systems for chemoselective and stereoselective oxidations. Eur. J. Org. Chem. 2015, 2015, 915-932. [CrossRef]

3. De Gonzalo, G.; Fraaije, M.W. Recent developments in flavin-based catalysis. ChemCatChem 2013, 5, 403-415. [CrossRef]

4. Zelenka, J.; Hartman, T.; Klímová, K.; Hampl, F.; Cibulka, R. Phase-transfer catalysis in oxidations based on the covalent bonding of hydrogen peroxide to amphiphilic flavinium salts. ChemCatChem 2014, 6, 2843-2846. [CrossRef]

5. Imada, Y.; Kitagawa, T.; Iwata, S.; Komiya, N.; Naota, T. Oxidation of sulfides with hydrogen peroxide catalyzed by synthetic flavin adducts with dendritic bis(acylamino)pyridines. Tetrahedron 2014, 70, 495-501. [CrossRef]

6. Ménová, P.; Dvořáková, H.; Eigner, V.; Ludvík, J.; Cibulka, R. Electron-deficient alloxazinium salts: Efficient organocatalysts of mild and chemoselective sulfoxidations with hydrogen peroxide. Adv. Synth. Catal. 2013, 355, 3451-3462. [CrossRef]

7. Jurok, R.; Hodačová, J.; Eigner, V.; Dvořáková, H.; Setnička, V.; Cibulka, R. Planar chiral flavinium salts: Synthesis and evaluation of the effect of substituents on the catalytic efficiency in enantioselective sulfoxidation reactions. Eur. J. Org. Chem. 2013, 2013, 7724-7738. [CrossRef]

8. Imada, Y.; Takagishi, M.; Komiya, N.; Naota, T. Oxidation of sulfides with hydrogen peroxide catalyzed by vitamin B2 derivatives. Synth. Commun. 2013, 43, 3064-3071. [CrossRef]

9. Shinkai, S.; Yamaguchi, T.; Manabe, O.; Toda, F. Enantioselective oxidation of sulphides with chiral 4a-hydroperoxyflavin. J. Chem. Soc. Chem. Commun. 1988, 1399-1401. [CrossRef]

10. Huijbers, M.M.E.; Montersino, S.; Westphal, A.H.; Tischler, D.; van Berkel, W.J.H. Flavin dependent monooxygenases. Arch. Biochem. Biophys. 2014, 544, 2-17. [CrossRef] [PubMed]

11. Forneris, F.; Heuts, D.P.H.M.; Delvecchio, M.; Rovida, S.; Fraaije, M.W.; Mattevi, A. Structural analysis of the catalytic mechanism and stereoselectivity in streptomyces coelicolor alditol oxidase. Biochemistry 2008, 47, 978-985. [CrossRef] [PubMed]

12. Matsui, T.; Dekishima, Y.; Ueda, M. Biotechnological production of chiral organic sulfoxides: Current state and perspectives. Appl. Microbiol. Biotechnol. 2014, 98, 7699-7706. [CrossRef] [PubMed]

13. Orru, R.; Dudek, H.M.; Martinoli, C.; Torres Pazmiño, D.E.; Royant, A.; Weik, M.; Fraaije, M.W.; Mattevi, A. Snapshots of enzymatic Baeyer-Villiger catalysis: Oxygen activation and intermediate stabilization. J. Biol. Chem. 2011, 286, 29284-29291. [CrossRef] [PubMed] 
14. Rioz-Martinez, A.; Kopacz, M.; de Gonzalo, G.; Torres Pazmino, D.E.; Gotor, V.; Fraaije, M.W. Exploring the biocatalytic scope of a bacterial flavin-containing monooxygenase. Org. Biomol. Chem. 2011, 9, 1337-1341. [CrossRef] [PubMed]

15. Colonna, S.; Sordo, S.D.; Gaggero, N.; Carrea, G.; Pasta, P. Enzyme-mediated catalytic asymmetric oxidations. Heteroat. Chem. 2002, 13, 467-473. [CrossRef]

16. Holland, H.L. Biotransformation of organic sulfides. Nat. Prod. Rep. 2001, 18, 171-181. [CrossRef] [PubMed]

17. Breslow, R.; Dong, S.D. Biomimetic reactions catalyzed by cyclodextrins and their derivatives. Chem. Rev. 1998, 98, 1997-2012. [CrossRef] [PubMed]

18. Marchetti, L.; Levine, M. Biomimetic catalysis. ACS Catal. 2011, 1, 1090-1118. [CrossRef]

19. Takahashi, K. Organic reactions mediated by cyclodextrins. Chem. Rev. 1998, 98, 2013-2034. [CrossRef] [PubMed]

20. Kanagaraj, K.; Suresh, P.; Pitchumani, K. Per-6-amino- $\beta$-cyclodextrin as a reusable promoter and chiral host for enantioselective Henry reaction. Org. Lett. 2010, 12, 4070-4073. [CrossRef] [PubMed]

21. Shen, H.-M.; Ji, H.-B. Amino alcohol-modified $\beta$-cyclodextrin inducing biomimetic asymmetric oxidation of thioanisole in water. Carbohydr. Res. 2012, 354, 49-58. [CrossRef] [PubMed]

22. Suresh, P.; Pitchumani, K. Per-6-amino- $\beta$-cyclodextrin catalyzed asymmetric michael addition of nitromethane and thiols to chalcones in water. Tetrahedron Asymmetry 2008, 19, 2037-2044. [CrossRef]

23. Chan, W.-K.; Yu, W.-Y.; Che, C.-M.; Wong, M.-K. A cyclodextrin-modified ketoester for stereoselective epoxidation of alkenes. J. Org. Chem. 2003, 68, 6576-6582. [CrossRef] [PubMed]

24. Rousseau, C.; Christensen, B.; Bols, M. Artificial epoxidase II. Synthesis of cyclodextrin ketoesters and epoxidation of alkenes. Eur. J. Org. Chem. 2005, 2005, 2734-2739. [CrossRef]

25. Schlatter, A.; Kundu, M.K.; Woggon, W.-D. Enantioselective reduction of aromatic and aliphatic ketones catalyzed by ruthenium complexes attached to $\beta$-cyclodextrin. Angew. Chem. Int. Ed. 2004, 43, 6731-6734. [CrossRef] [PubMed]

26. Ye, H.P.; Tong, W.; D'Souza, V.T. Efficient catalysis of a redox reaction by an artificial enzyme. J. Am. Chem. Soc. 1992, 114, 5470-5472. [CrossRef]

27. Ye, H.; Tong, W.; D'Souza, V.T. Flavocyclodextrins as artificial redox enzymes. Part 4. Catalytic reactions of alcohols, aldehydes and thiols. J. Chem. Soc. Perkin Trans. 2 1994, 2431-2438. [CrossRef]

28. D'Souza, V.T. Modification of cyclodextrins for use as artificial enzymes. Supramol. Chem. 2003, 15, $221-229$. [CrossRef]

29. Mojr, V.; Herzig, V.; Buděšínský, M.; Cibulka, R.; Kraus, T. Flavin-cyclodextrin conjugates as catalysts of enantioselective sulfoxidations with hydrogen peroxide in aqueous media. Chem. Commun. 2010, 46, 7599-7601. [CrossRef] [PubMed]

30. Mojr, V.; Buděšínský, M.; Cibulka, R.; Kraus, T. Alloxazine-cyclodextrin conjugates for organocatalytic enantioselective sulfoxidations. Org. Biomol. Chem. 2011, 9, 7318-7326. [CrossRef] [PubMed]

31. Hartman, T.; Herzig, V.; Buděšínský, M.; Jindřich, J.; Cibulka, R.; Kraus, T. Flavin-cyclodextrin conjugates: Effect of the structure on the catalytic activity in enantioselective sulfoxidations. Tetrahedron Asymmetry 2012, 23, 1571-1583. [CrossRef]

32. Kolb, H.C.; Finn, M.G.; Sharpless, K.B. Click chemistry: Diverse chemical function from a few good reactions. Angew. Chem. Int. Ed. 2001, 40, 2004-2021. [CrossRef]

33. Amblard, F.; Cho, J.H.; Schinazi, R.F. Cu(I)-catalyzed Huisgen azide-alkyne 1,3-dipolar cycloaddition reaction in nucleoside, nucleotide, and oligonucleotide chemistry. Chem. Rev. 2009, 109, 4207-4220. [CrossRef] [PubMed]

34. Faugeras, P.-A.; Boëns, B.; Elchinger, P.-H.; Brouillette, F.; Montplaisir, D.; Zerrouki, R.; Lucas, R. When cyclodextrins meet click chemistry. Eur. J. Org. Chem. 2012, 2012, 4087-4105. [CrossRef]

35. Xu, L.; Li, Y.; Li, Y. Application of "click" chemistry to the construction of supramolecular functional systems. Asian J. Org. Chem. 2014, 3, 582-602. [CrossRef]

36. Melone, L.; Petroselli, M.; Pastori, N.; Punta, C. Functionalization of cyclodextrins with $N$-hydroxyphthalimide moiety: A new class of supramolecular pro-oxidant organocatalysts. Molecules 2015, 20, 15881-15892. [CrossRef] [PubMed]

37. Carroll, J.B.; Jordan, B.J.; Xu, H.; Erdogan, B.; Lee, L.; Cheng, L.; Tiernan, C.; Cooke, G.; Rotello, V.M. Model systems for flavoenzyme activity: Site-isolated redox behavior in flavin-functionalized random polystyrene copolymers. Org. Lett. 2005, 7, 2551-2554. [CrossRef] [PubMed] 
38. Subramani, C.; Yesilbag, G.; Jordan, B.J.; Li, X.; Khorasani, A.; Cooke, G.; Sanyal, A.; Rotello, V.M. Recognition mediated encapsulation and isolation of flavin-polymer conjugates using dendritic guest moieties. Chem. Commun. 2010, 46, 2067-2069. [CrossRef] [PubMed]

39. Petter, R.C.; Salek, J.S.; Sikorski, C.T.; Kumaravel, G.; Lin, F.T. Cooperative binding by aggregated mono-6-(alkylamino)- $\beta$-cyclodextrins. J. Am. Chem. Soc. 1990, 112, 3860-3868. [CrossRef]

40. Donnelly, P.S.; Zanatta, S.D.; Zammit, S.C.; White, J.M.; Williams, S.J. “Click” cycloaddition catalysts: Copper(I) and copper(II) tris(triazolylmethyl)amine complexes. Chem. Commun. 2008, 2459-2461. [CrossRef] [PubMed]

41. Bach, R.D. General and Theoretical Aspects of the Peroxide Group, 2006; Rappoport, Z., Ed.; John Wiley \& Sons Ltd.: Chichester, UK, 2006; Volume 2, pp. 1-92.

42. De Gonzalo, G.; Torres Pazmiño, D.E.; Ottolina, G.; Fraaije, M.W.; Carrea, G. 4-Hydroxyacetophenone monooxygenase from pseudomonas fluorescens ACB as an oxidative biocatalyst in the synthesis of optically active sulfoxides. Tetrahedron Asymmetry 2006, 17, 130-135. [CrossRef]

43. Rioz-Martínez, A.; de Gonzalo, G.; Pazmiño, D.E.T.; Fraaije, M.W.; Gotor, V. Enzymatic synthesis of novel chiral sulfoxides employing Baeyer-Villiger monooxygenases. Eur. J. Org. Chem. 2010, 2010, 6409-6416. [CrossRef]

44. Spinella, A.; Caruso, T.; Martino, M.; Sessa, C. Synthesis of aplyolide A, ichthyotoxic macrolide isolated from the skin of the marine mollusk Aplysia depilans. Synlett 2011, 12, 1971-1973.

45. Smith, L.H. Tetrahydrofurfuryl bromide. Org. Synth. 1943, 23, 88-89.

46. Jones, E.R.H.; Eglinton, G.; Whiting, M.C. 4-Pentyn-1-ol. Org. Synth. 1963, 4, 755. [CrossRef]

47. Rodríguez Rivero, M.; Alonso, I.; Carretero, J.C. Vinyl sulfoxides as stereochemical controllers in intermolecular Pauson-Khand reactions: Applications to the enantioselective synthesis of natural cyclopentanoids. Chem.-Eur. J. 2004, 10, 5443-5459. [CrossRef] [PubMed]

48. Cibulka, R.; Baxová, L.; Dvořáková, H.; Hampl, F.; Ménová, P.; Mojr, V.; Plancq, B.; Sayin, S. Catalytic effect of alloxazinium and isoalloxazinium salts on oxidation of sulfides with hydrogen peroxide in micellar media. Collect. Czechoslov. Chem. Commun. 2009, 74, 973-993. [CrossRef]

49. Nandwana, V.; Samuel, I.; Cooke, G.; Rotello, V.M. Aromatic stacking interactions in flavin model systems. Acc. Chem. Res. 2012, 46, 1000-1009. [CrossRef] [PubMed]

Sample Availability: Samples of the compounds $\mathbf{8}$ and $\mathbf{9}$ are available from the authors.

(C) 2015 by the authors; licensee MDPI, Basel, Switzerland. This article is an open access article distributed under the terms and conditions of the Creative Commons by Attribution (CC-BY) license (http://creativecommons.org/licenses/by/4.0/). 